OPERANT LEARNING

\section{Automatic operant conditioning}

Erskin, A., Bus, T., Herb, J.T., Schaefer, A.T.

PLoS One 14, e0211571 (2019)

To increase the throughput of training and testing multiple mice on an operant conditioning task while keeping handling to a minimum, researchers from the Francis Crick Institute in London have developed the 'AutonoMouse' house. It's a two-story home cage that can house up to 25 radio-frequency identification tagged mice at a time. The mice eat, sleep, and recreate on the lower level and ascend upstairs to perform an operant conditioning task to receive water.

Humans need only be involved for health checks and cage cleanings.

The researchers demonstrate the AutonoMouse with olfactory discrimination tasks to automatically test mice before and after experimentally induced olfactory bulb lesions, which were shown to impair odor discrimination. The AutonoMouse design is open source, and more details can be found in the paper and on GitHub.

$E P N$

https://doi.org/10.1038/s41684-019-0301-2

\section{NEUROSCIENCE}

\section{Towards a marmoset brain map}

Lin, M.K. et al. elife 8, e40042 (2019)

The marmoset (Callithrix jacchus) is an increasingly popular animal model for neuroscience research. The animals are smaller and easier to work with than macaques, but more closely related to humans than rodents. Efforts to produce a complete brain connectivity map for the marmoset, akin to what's available for mouse, are underway. Researchers at the RIKEN Center for Brain Science and Cold Spring Harbor Laboratory recently published details of their high-throughput neurohistological pipeline.

They establish a grid-based tracer injection strategy to map mesoscale neural connections in the marmoset brain. Sections of brain are then cryo-stained, and a computer program processes $3 \mathrm{D}$ images from the tracer, staining, and in vivo MRI data. Completed images so far can be viewed at marmoset.brainarchitecture.org. A map of the entire brain is expected by 2024 . EPN

\section{GENOMICS}

\section{Beneath the icefish}

Kim, B. et al. Nat. Ecol. Evol. 3, 469-478 (2019)

Antarctic icefishes have evolved some unique adaptations to their extreme polar environments. Notably, the different species of notothenioid fishes are 'whiteblooded'-in order to survive temperatures as low as $-1.9^{\circ} \mathrm{C}$, they express antifreeze glycoproteins and lack red blood cells. They could thus be useful models to understand pathological conditions in humans, such as anemia.

To understand the animals and their adaptations better, a team of researchers recently completed a highquality genome assembly and linkage map for the Antarctic blackfin icefish, Chaenocephalus aceratus. Though in many places the genome of the icefish was highly conserved with red-blooded relatives, the results reveal expansions in genes for antifreeze proteins and those that maintain cellular redox states in the fish. The icefish also lack several genes that regulate circadian rhythms.

https://doi.org/10.1038/s41684-019-0303-0

NEUROSCIENCE

\section{Polymer arrays for chronic recording}

Chung, J.E. et al. Neuron 101, 21-31 (2019)

Electrophysiology systems for use in rodents in the past were often limited: they could record the activity of many single neurons but for only a short time, while recording longer often meant giving up some spatial resolution. But a new tool from researchers at the University of California, San Francisco improves both the temporal and spatial performance of a single set up. The team built an electrophysiology system from polymer probes (more biocompatible than microwires) that are arranged into 16 modules that can be placed throughout different regions of the brain. The modules are forked, and each fork can record activity from 4 locations, adding up to 1,024 recording channels. The system recorded electrophysiology data from individual neurons in freely behaving rats for up to 160 days.

https://doi.org/10.1038/s41684-019-0304-z

Alexandra Le Bras and Ellen P. Neff

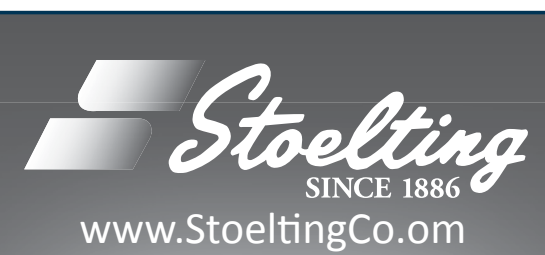

Lab Animal Identification SMALL ANIMAL EARTAGS AND MARKERS
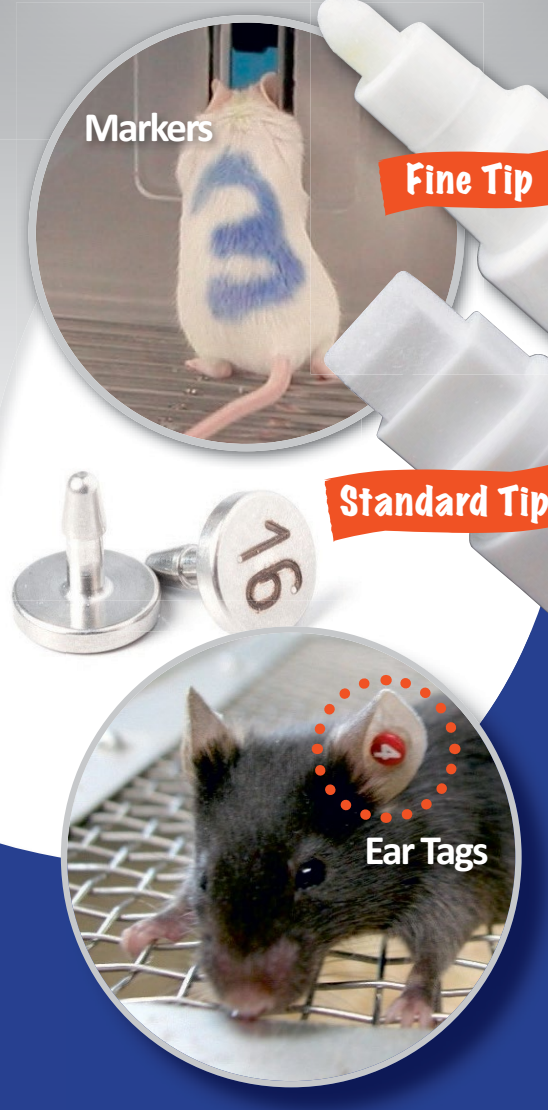

Ear Tag and Marker Features:

- Quick and easy identification

- Long-lasting

- Nontoxic, non-hazardous

- Multiple colors options available

Samples now available,

Contact Stoelting for

more details!

info@stoeltingco.com 